\title{
EL DÉMARCHE ERGOLÓGICO EN EL ANÁLISIS DE LA ACTIVIDAD DE TRABAJO DEL PROFESOR UNIVERSITARIO
}

DOI: https://doi.org/10.35699/2237-5864.2018.2210

\author{
Daniel Fabián Roca Flores Pinto ${ }^{1}$ \\ Daisy Moreira Cunha² \\ Rogério Cunha Campos ${ }^{2}$
}

\section{RESUMEN}

La presente comunicación científica es parte de una tesis de doctorado, que aborda desde la perspectiva ergológica la actividad de trabajo del profesor universitario situado en la sala de aula, en una universidad de carácter comunitario, originaria de un movimiento estudiantil, inserta en un contexto universitario regulado por valores mercantiles que impactan en la docencia como bien público, silenciando el potencial transformador de los saberes producidos en las situaciones de trabajo. Esta investigación de abordaje clínico y de carácter cualitativo experimental, problematiza el cotidiano universitario permeado por una concepción tradicional del trabajo, ampliando su discusión, desvelando los patrimonios creados y recreados en ese medio; los usos de sí; los saberes operantes en las situaciones de trabajo y las elecciones de valor del profesor, como elementos que dan sentido y significado sobre su trabajo, colocando abiertas las posibilidades para su transformación.

Palabras claves: Démarche ergológico. Actividad del profesor universitario. Universidad Peruana.

\footnotetext{
${ }^{1}$ Universidad de Ciencias y Humanidades, Lima, Perú.

${ }^{2}$ Faculdade de Educação, Universidade Federal de Minas Gerais, Belo Horizonte, MG, Brasil.
} 


\title{
O DÉMARCHE ERGOLÓGICO NA ANÁLISE DA ATIVIDADE DE TRABALHO DO PROFESSOR UNIVERSITÁRIO
}

\author{
Daniel Fabián Roca Flores Pinto \\ Daisy Moreira Cunha \\ Rogério Cunha Campos
}

\section{RESUMO}

Este artigo é parte de uma tese de doutorado que aborda, a partir da perspectiva ergológica, a atividade de trabalho do professor universitário em sala de aula, em uma universidade de caráter comunitário, originária do movimento estudantil, inserida no contexto universitário regulado por valores mercantis que impactam a docência como um bem público e silenciam o potencial transformador dos saberes produzidos nas situações de trabalho. Essa pesquisa, de abordagem clínica e caráter qualitativo experimental, problematiza o cotidiano universitário, desvelando os patrimônios criados e recriados nesse meio e os seus usos, os saberes de valores operantes nas situações de trabalho e as eleições de valor assumidas pelo professor, como elementos que oferecem sentido e significado sobre seu trabalho e que colocam em aberto as possibilidades de sua transformação.

Palavras-chave: Démarche ergológico. Atividade do professor universitário. Universidade Peruana. 


\title{
THE ERGOLOGICAL DÉMARCHE IN THE ANALYSIS OF THE WORK ACTIVITY OF THE UNIVERSITY TEACHER
}

Daniel Fabián Roca Flores Pinto

Daisy Moreira Cunha

Rogério Cunha Campos

\begin{abstract}
The present article is part of a doctoral thesis, which approaches, from the ergological perspective, the work activity of the university professor located in the classroom, in a community university, originated in a student movement, inserted in a university context regulated by mercantile values that impact on teaching as a public good and silence the transforming potential of the knowledge produced in work situations. This investigation, of clinical approach and of qualitative experimental character, problematizes an university environment permeated by a traditional conception of work, expanding its discussion, revealing the patrimonies created and recreated in that environment and it's uses, the knowledges operative in situations of work and the elections of value of professors, as elements that give sense and meaning on their work, placing open the possibilities for their transformation.
\end{abstract}

Keywords: Ergological démarche. University teacher activity. Peruvian university.

Received on: 11/07/2017

Approved on: 20/04/2018 


\section{CONSIDERACIONES PRELIMINARES}

Las investigaciones en el Perú sobre la educación superior, sus instituciones y específicamente el trabajo del profesor universitario vienen ganando un mayor interés en los diferentes espacios, principalmente en el ámbito académico. No obstante, aún se caracterizan por la dispersión de los datos analizados y el carácter reservado en el tratamiento de estas temáticas por parte de las universidades, sobresaliendo en estas producciones el uso de perspectivas teóricas que enfocan principalmente los aspectos organizacionales, económicos y productivos; dejándose ausente en estos estudios a los sujetos, sus prácticas y producciones, considerados como aspectos constitutivos para repensar en las transformaciones necesarias para este importante sector educativo (ROCA, 2017).

Según Pásara (2013) y Rodríguez (2007) el contexto universitario peruano está mercantilizado, estando actualmente regulado por un marco normativo atravesado por principios mercantiles que fortalecen las relaciones de dar-pagar, convirtiendo al estudiante en cliente y al conocimiento del profesor en una mercancía, con lo cual se cosifica la docencia, pero también se incorpora sin cuestionamiento en las Instituciones de Educación Superior (IES) sean de naturaleza pública o privada un modelo de gestión gerencial, orientado al cumplimiento de parámetros e indicadores impuestos por el nuevo órgano nacional de control, con la finalidad de obtener y/o mantener su licenciamiento, necesario para continuar funcionando y captando más estudiantes.

Esta nueva regulación y modelo de gerenciamiento vigente termina por influenciar e impactar en el colectivo de profesores, fragilizando la dimensión profesional del docente, convirtiendo al profesor universitario en un prestador de servicios, sometido a trabajar bajo un régimen flexible de contratación parcial por semestre, laborando en varios turnos, enseñando diversas disciplinas que no necesariamente domina y siendo pagos sólo por las horas dedicadas a la enseñanza. Dicha situación, los coloca en la búsqueda permanente de empleo, pues no está garantizada su continuación para el siguiente semestre.

Por otra parte, esta forma de organización del trabajo del profesor, coloca al coordinador académico como el administrador del servicio prestado, como mercancía al conocimiento del profesor y al estudiante como cliente. La fidelización de este último está garantizada mediante la incorporación de procesos estandarizados y homogéneos de las prácticas docentes que 
puedan ser monitoreadas y evaluadas por la aplicación y la aprobación de encuestas de satisfacción del servicio por parte del cliente alumno.

En este medio, las normas que giren la comprensión sobre el trabajo del profesor y por otro lado las perspectivas que orientan las investigaciones y estudios realizados, dejan fuera de escena al sujeto de la actividad, silenciando sus subjetividades y singularidades, desconsiderando el potencial y la riqueza de los patrimonios construidos y reconstruidos en las situaciones de trabajo y con ello, las posibilidades para mejorar sus condiciones y transformaciones de sus procesos de trabajo (SCHWARTZ; DURRIVE, 2010).

La ruptura con estas formas hegemónicas y limitantes de comprender y abordar las cuestiones sobre la educación superior, sus instituciones y el trabajo del profesor universitario, posibilitaron incorporar otras y nuevas perspectivas que amplían el debate y su discusión. Además, favorece una comprensión mayor sobre la condición y desenvolvimiento de sujetos vivos que realizan diálogos dialécticos sobre la estructura social, sus normas, sus prácticas y las complejas relaciones de carácter social, cultural y político que se establecen con los diversos colectivos presentes en los espacios educativos.

En ese sentido, proponemos abordar el trabajo del profesor universitario mediante una perspectiva ergológica que permite profundizar en el nivel micro de su actividad y analizar como las normas institucionales permean su trabajo, las relaciones que él establece con el conocimiento, con su práctica y con los diversos colectivos con quien interactúa. Así como la creación y recreación de sus propias normas para cumplir con los objetivos impuestos y con los que el profesor así mismo se impone.

La perspectiva ergológica nace buscando comprender el camino tortuoso del trabajo industrial y de los servicios de las grandes ciudades, en que hombres y mujeres se forjan interactuando con las situaciones, las reglas, las coyunturas que vivencían, así como los imaginarios simbólicos que se van configurando en la propia sociedad, ejerciendo menor o mayor presión en los sujetos productores y sobre su trabajo.

La ergología, término que proviene del griego ergo (acción, trabajo), que Schwartz integra a los aportes de diversas disciplinas que estudian el trabajo (Psicología, Psicodinámica del trabajo, Sociología del trabajo, Clínica de la actividad y la Ergonomía de la actividad), permite hacer lecturas antropológicas sobre el trabajo, colocando en escena situaciones laborales y 
sujetos vivos, representados y haciendo uso de sí por otros, pero también por sí mismos. Esos encuentros esclarecen diversos aspectos particulares de la experiencia del trabajo, resultante del ejercicio profesional.

De esa manera, se puede pensar en las articulaciones del trabajo con la vida, con la formación de identidades, de competencias profesionales, así como de los laberintos del ser en los oficios laborales, ampliando hacia una visión más plural sobre la experiencia de los trabajadores en nuestros análisis.

El entendimiento de la complejidad de esa experiencia posibilita la comprensión de los debates de normas producidos, las renormalizaciones que se llevan a cabo desvelándose los saberes y valores operantes en las situaciones de trabajo. Ampliándose los espacios para discutir y repensar el trabajo, procurándose su transformación y mejoría, tornándolo más humano y reconociendo la creación de patrimonios que van más allá de valores sólo económicos.

En ese sentido, la ergología se define como un démarche de producción de saberes sobre el trabajo, sin que con ello se tenga que plantear como un único método de trabajo definido, pudiéndose tener un conjunto de especificaciones y orientaciones metodológicas desde que se tenga en cuenta la lógica propuesta por su dispositivo de producción de conocimiento. Tal dispositivo no debiera ser entendido como arbitrariedad o totalidad, sino como un punto de partida, dado que posibilita un análisis pluridisciplinar de la actividad, con lo cual se confrontan saberes académicos y saberes desarrollados por los trabajadores en su cotidiano. Así, el dispositivo ergológico incluye varios métodos, los cuales permiten la producción de conocimientos de situaciones reales empleando instrumentos de recojo de datos como observaciones, entrevistas, conversas u otras técnicas, objetivándose siempre la actividad humana.

La perspectiva de la ergología y su démarche, es elegida para esta investigación, al encontrar como posibilidad para estudiar al trabajador en el nivel micro de la actividad y no sólo como un sujeto que lleva a cabo una función, ejecutando tareas o cumpliendo normas; sino comprendiendo al trabajo como un espacio vivo, como lugar de producción de saberes y de circulación de valores no sólo económicos (SCHWARTZ, 2004). También se hace necesario incorporar al profesor trabajador como un sujeto ético-político, histórico, sociocultural y epistemológico (CUNHA, 2010) procurando la comprensión de las dificultades propias de su trabajo, de los saberes y valores producidos, desvelando ese trabajo a partir del propio actor. 
Para ello, es necesario sumergirnos con él en su actividad y profundizarnos en su singularidad y en las diversas coyunturas (político-sociales y económicas) que lo influyen.

El medio en el cual está inserto el profesor universitario peruano se representa como una sociedad democrática, donde existe una polaridad problemática de ambivalencia y estreches entre sus polos que conforman el llamado "esquema tripolar". Con este dispositivo, realizamos el análisis de los tres polos ergológicos que configuran la sociedad peruana.

Así, el polo del bien común, en el cual un bien público como la educación viene siendo dimensionado bajo la anuencia del polo del derecho, pero bajo normas que establecen un contexto de mercantilización en los niveles educacionales Básico y Superior (CHIROKE, 2010).

Estos niveles educacionales están permeados por valores del mercado, en los que se resaltan valores económicos sobre su trabajo y están regulados por un órgano estatal de carácter privado mercantil que establece arbitrariamente el cumplimiento de parámetros y estándares afines a la gestión empresarial. Con esos estándares, el oficio del profesor se ha reconfigurado al de un prestador de servicios, colocando su actividad como privada (SGUISSARDI, 2005), con la cual, se flexibiliza su condición al trabajador por horas, con la cual, son establecidos los nuevos regímenes de contratación parcial para la conformación del cuerpo docente en las universidades públicas y privadas, reduciendo su dimensión profesional a sólo enseñantes, ejerciendo un disciplinamiento mediante el cumplimiento de dispositivos burocráticos.

Esta situación coloca al profesional docente en permanentemente búsqueda de empleo, participando de concursos para la obtención de vacantes y preocupados por donde enseñarán el siguiente semestre (CHOMSKY, 2014), reduciéndose sus posibilidades para conformar colectivos y tener espacios para discutir sobre su trabajo, invisibilisándose con ello los patrimonios creados y recreados en las situaciones de trabajo. Siendo necesaria su comprensión para la transformación y mejoría de su trabajo, así como para comprender que su trabajo no es sólo ejecutar tareas o cumplir normas. Comprendiendo así, al trabajo como espacio vivo, en donde saberes y valores son movilizados no sólo con una orientación económica, sino también ética-política, histórica, sociocultural y epistemológica, incorporando al propio sujeto en la proposición de estas acciones.

En el contexto señalado anteriormente, el estudio del trabajo bajo el referencial de la ergología, nos provoca un "malestar intelectual" (SCHWARTZ, 2000, p. 44) al admitir que generalidades 
y modelizaciones deben ser siempre re-apreciadas y por tanto, considerar al trabajo como "materia extraña", es decir, que a priori no podemos definirlo, siendo imposible conocerlo si estamos fuera de él.

Schwartz (2003) propone que como investigadores nos coloquemos en el aprendizaje junto a los hombres y mujeres que trabajan, intentando comprender lo que está sucediendo y las relaciones que ahí se establecen, considerando el carácter enigmático de las situaciones de trabajo, por tanto, conceptualmente, no hay forma de anticiparlo, al ser el trabajo considerado desde esta perspectiva como un evento que convoca a quien lo realiza en su totalidad y que está más allá de una observación sobre la actividad, donde "lo esencial de la actividad de trabajo no se ve" (SCHWARTZ, 2011, p. 133).

\section{EL ENCUENTRO CON LA ACTIVIDAD: SUJETOS PARTICIPANTES Y METODOLOGÍA}

Al establecer como objetivo la comprensión y análisis de la actividad del profesor universitario en sala de aula, reconocemos que la aproximación sobre la actividad, implica contar con participantes voluntarios e informados sobre el proceso de la investigación y pertenecientes a una institución educativa singular en sus fines y carácter social, pero también por su origen e historia, su propuesta educativa y política, así como por sus colectivos que la constituyen.

En ese sentido, son elegidos tres profesores formadores de ingenieros, quienes participan voluntariamente, siendo preservadas sus identidades a través del uso de siglas para cada profesor (Profesor 1: P1; Profesor 2: P2; Profesor 3: P3), elegidos bajo los criterios de similitud en la actividad que realizan, tiempo en la docencia, régimen contractual vigente, formación y por pertenecer a una universidad no estatal sin fines de lucro, inserta en un contexto mercantil anteriormente explicado.

El profesor participante de la investigación se caracteriza por dedicarse desde hace diez años a la docencia universitaria, contratado como enseñante, bajo un régimen parcial de 20 horas, sin dedicación exclusiva, formado en ingeniería y/o áreas afines en una universidad pública y perteneciente a la Facultad de Ciencias e Ingeniería.

La Facultad de Ciencias e Ingeniería, institución a la cual el profesor está vinculado, presenta el mayor número de estudiantes y profesores de una universidad peruana no estatal, sin fines 
de lucro, ubicada en la periferia de Lima Norte, de carácter comunitario y originario de un movimiento estudiantil universitario en los años sesenta.

Las características singulares de esta universidad donde situamos al profesor, inicialmente organizada como una asociación de profesores, quienes convocaron a importantes representantes de la educación peruana y de las otras áreas del conocimiento, para la elaboración de un proyecto educativo orientado a la formación integral de las camadas populares migrantes del interior del país, asentados en las zonas periféricas y aledañas a la ubicación de campus universitario, pero también relacionadas con su historia y origen.

En ese medio de características singulares inserto en un contexto de universidades mercantiles, el método y los procedimientos empleados para el estudio del trabajo como actividad humana están asociados con la perspectiva ergológica, optándose por un abordaje clínico, al considerar el sentido enigmático y singular de aproximación al trabajo del profesor, bajo un régimen de coproducción y colaboración, envolviendo, la racionalidad de la acción y el medio científico, orientado a la producción y creación de nuevos territorios existenciales (FIGUEREIDO \& ATHAYDE, 2004).

Serán consideradas las verbalizaciones de los propios profesores sobre su trabajo, quienes participan en la comprensión de aquello que es visible y no visible sobre su trabajo, los compromisos que asumen, las evaluaciones que realizan sobre aquello que hacen y cómo se movilizan para salir bien, aportando aquel conocimiento infinitesimal de su oficio y de la dimensión gestionaria que aparece en su hacer situado en sala de aula, comprendido este, como el lugar donde se interrelaciona lo institucional con lo cotidiano. Los diálogos son producidos a partir de provocaciones que el investigador establece con cada profesor, mediante el uso de tres procedimientos metodológicos que se articulan entre sí: la entrevista semi estructurada (ROCHA et al., 2004), el método de la instrucción al sósia (ODDONE et al., 1986; CLOT, 2010) y el método de la autoconfrontación simple (FAïTA, 1997).

La entrevista semi estructurada empleada de forma individual con cada profesor, es también la creación de un espacio de escucha al trabajador y empleada como un dispositivo enunciativo de producción de textos y de circulación de determinados discursos, que son recortados conforme los objetivos que se persiguen, procurando mutilar lo menos posible la actividad y buscando ahondar en su dimensión personal y profesional. 
Para generar los diálogos iniciales con el profesor empleamos una matriz de temas conformada por cinco ejes: (i) trayectoria, expectativas y perspectivas sobre su trabajo; (ii) relaciones con otros colectivos; (iii) impacto de su trabajo en su vida-salud; (iv) organización de la disciplina que conduce y $(v)$ valoración institucional sobre su trabajo. Siendo estos ejes elegidos entre otros, por aportar elementos claves en las cuestiones relacionadas con el sentido y significado de su trabajo para el profesor participante así como los modos de operación creados por él, para realizar su actividad y salir bien.

Estos ejes son profundizados mediante un conjunto de preguntas que se amplían conforme ocurran los encuentros y por los andamiajes que se van encontrando al conocer la situación de trabajo en que está cada profesor. Así, se construye un texto por cada profesor, con el cual, vamos confeccionando un mapa preliminar sobre él, su trabajo y las características de la institución en donde trabaja.

El análisis de la entrevista desvela como el carácter social de esta IES posibilita que el profesor genere nuevas negociaciones con los estudiantes, con la coordinación académica, renormalizando las normas antecedentes que intentan anticipar su actividad; asimismo se constata que lo institucional permea la actividad del docente y que su trabajo no es apenas dirigido a los alumnos sino también a la IES donde trabaja (AMIGUES, 2004).

Su trabajo es una actividad socialmente situada y mediada por los objetivos que constituyen un sistema, cuyo objeto es la creación y organización de un medio que sea favorable al desenvolvimiento de capacidades de los estudiantes y aprendizajes, en donde lo comunitario es comprendido como un privado social (QUIJANO, 2014). Esto no es sólo una característica del ámbito de lo institucional, sino que esta inserida en el sujeto singular, en la construcción subjetiva de su actividad, sus vivencias y en sus experiencias, así como por sus elecciones de valor, donde la realizar la docencia le implica vincularse y comprometerse con un tipo de bien público, entendido por él, como un hacer vehiculado para que el estudiante al aprender su disciplina, construya un mayor interés y sentido sobre lo que estudia, involucrándolo en ese quehacer social y hacia lo colectivo.

El método de la Instrucción Al Sósia (IAS) se emplea como una estrategia de aproximación de la actividad de cada profesor en sala de aula, asumiendo el investigador el papel de sósia (doble) que sustituiría al profesor (instructor) en un día de clase, provocándose un dialogo inicial mediante: "supongamos que yo sea su sósia y mañana me encuentre en la situación de 
sustituirlo en su trabajo: ¿qué instrucciones me debería transmitir, a fin de que nadie se diera cuenta de esta situación?".

El resultado de este primer encuentro, no consiguió, el dialogo esperado con el profesor, quien no reconoció en el sósia la formación idónea para reemplazarlo d resaltando las especificidades propias de la disciplina que él enseña y la experiencia necesaria para abordarla.

Este hecho nos movilizó en la necesidad de colocar una tercera persona como figura, sin que con ello se afecte el método en sí. Recomenzándose el diálogo: "supongamos que un colega de su entera confianza, formación y experiencia tenga que sustituirlo, que instrucciones daría para él, a fin que no se note esta situación?

Esta reformulación propició un diálogo fecundo entre ambos y posibilitó que el profesor nos hable sobre aquello que implica realizar la clase, identificándose así, que esta empieza y continua fuera de este espacio, que la misma es un encuentro único, que no se repite y que también es diversa (diferentes alumnos e historias de vida personales), llena de situaciones (siempre ocurre alguna novedad en clase) que implica movilizar y traer sus experiencias (estando presente lo que aprendió en otros espacios), dominios (formación y experiencia de vida) y enfrentar situaciones que se suscitan, en un aquí y ahora (con los alumnos, con la coordinación, con otros colectivos).

Este avance, desveló los desdoblamientos que el profesor realiza (trabajar en casa, entregar avances, cumplir procedimientos), el impacto de las normas (curriculares y administrativas) en sus vidas (la salud, su familia, en lo personal y lo profesional), pero también los márgenes que él encuentra para proponer y modificar (nuevas negociaciones y acuerdos se producen) y empezar a tener sentido para él sobre aquello que deja de hacer (elecciones y arbitrajes presentes).

Los diálogos fueron producidos voluntariamente y otras veces provocados, intentando llevar a la luz los debates de normas (confrontamientos), renormalizaciones (creación de sus propias normas) y valores allí presentes (arbitrajes y elecciones), elaborándose un texto escrito que nuevamente fue dialogado con el profesor en un segundo encuentro, constituyendo la Autoconfrontación Simple (ACS).

El análisis de las verbalizaciones producidas en la IAS retrata un camino de superación de múltiples dificultades que el profesor enfrenta en su cotidiano. Se pueden mencionar los 
diversos grupos de alumnos que conforman sus turnos y los otros lugares donde comparte la enseñanza, trabajos fuera de clase (consultorías), corrección de exámenes, trabajos, colocación de materiales en la intranet y otros procedimientos burocráticos que demandan esfuerzos y dedicación de tiempo que terminan por establecer nuevas condiciones de trabajo que tiene que aceptar para trabajar. No obstante, con la identificación con su actividad, el profesor crea maniobras necesarias para alcanzar su satisfacción al realizarlas. Estas maniobras, denominadas como modos de operación, son lo que nos aproximan al encuentro con la actividad del profesor, remitiéndonos a la conducción de sus clases en sala de aula, subyaciendo espacios que extrapolan el espacio físico (aula y laboratorio), la existencia de distintas formas de hacer las cosas (operar), saberes y valores dinamizados (elecciones y elementos empleados para enseñar) y los nuevos patrimonios (producciones de ese encuentro de la experiencia en situaciones de trabajo), presentes en la preparación de las clases, en el cumplimiento de los procedimientos académicos y curriculares, en las interacciones con los estudiantes en los turnos donde enseñan, en la evaluación arbitraria a la que son sometidos.

El método de Autoconfrontación Simple (ACS) no contemplo el uso del vídeo, al mantenerse el acuerdo previo con los profesores, de no ser filmados y fotografiados. De esta manera cada profesor fue abordado individualmente con la transcripción de las verbalizaciones producidas en la IAS, enfatizándose las situaciones, profundizándose en los detalles y en las reflexiones generadas.

Los textos presentados a cada profesor guardaron relación con la diversidad de alumnos que componen sus grupos y turnos, con la incorporación de discusiones sociales y políticas durante el desarrollo de contenidos técnicos, con los dispositivos burocráticos que los someten y con las condiciones y regímenes de trabajo impuestos por el sistema universitario actual.

Este procedimiento colocó sobre el tapete como su trabajo en sala de aula es un espacio emancipatorio y concomitante con los inicios de esta IES, el poder de hacer del docente y las posibilidades para que su práctica sea reconocida no apenas como un aspecto mercantil de fidelización, sino de transformación del proyecto educativo de esta universidad, así como para un entendimiento más amplio sobre su trabajo inserto en ese contexto.

El análisis posibilitó una aproximación sobre aquellas reservas de alternativas, como formas para afrontar su trabajo, así como las otras perspectivas para vivir y hacer en conjunto, 
manifestadas en sus actos cotidianos presentes en la gestión de su trabajo, las interacciones con el estudiante y colegas.

\section{CONSIDERACIONES FINALES}

Esta investigación pionera en el Perú en analizar el trabajo del profesor universitario desde la perspectiva ergológica, propone para el campo de estudio de la Educación Superior, repensar sobre el abordaje de este importante ámbito a través de perspectivas que exploren los diversos aspectos que las configuran y las dimensiones que constituyen al sujeto docente y la riqueza de sus patrimonios producidos en sus experiencias en situaciones de trabajo, ampliando nuevos espacios para discutir, plantear políticas y acciones que se orienten a la transformación y mejoría del trabajo, pero también para crear y ampliar la comprensión sobre los saberes y valores respecto al trabajo del profesor universitario en ese medio.

Asimismo, consideramos que este estudio ofrece elementos para que esta IES, donde se realizó la investigación, reflexione acerca del curso tomado respecto al desarrollo de su ideario inicial y como los valores mercantiles vigentes en el sistema universitario peruano han ido permeando y moldeando con una lógica distinta a lo que su colectivo institucional proyectó para esta universidad.

Al desvelar la actividad de trabajo del profesor participante de esta pesquisa, los debates de normas y las renormalizaciones producidas por sus usos de sí, recolocan su trabajo como un bien público, que revalora sus patrimonios creados, los sentidos y significados atribuidos sobre su trabajo, que presentan los trazos de un privado social amalgamado con el origen social y comunitario de esta IES, pudiendo ser estos movilizadores para la transformación y mejoría del trabajo del profesor.

Se constata a que las normas por si solas no aseguran la actividad, siendo las elecciones, los arbitrajes y la movilización de saberes adheridos a la actividad y manifestados en las situaciones de trabajo que configuran y colocan en emergencia el actual modelo de gestión y la apertura para espacios de discusión sobre otras formas gestionarias basadas en lo colectivo, solidario y recíproco como valores que posibiliten organizar el trabajo y el establecimiento de las relaciones. 
Las renormalizaciones producidas por el profesor evidencían los denominados "saberes valores", saberes heterogéneos que se enriquecen con los nuevos valores producidos, por las elecciones que el profesor decide tomar para enfrentar las diferentes situaciones que configuran su trabajo como profesor. Elecciones que dan vida a esas renormalizaciones y constatan, que algunas de ellas son obscuras y otras son conscientes, pudiendo ser algunas previsibles, pero no totalmente, pues estas sólo pueden ser entendidas en una situación de vida y de actividad, pues en ella, podemos comprender como él vive esa situación y lo que él puede hacer.

\section{REFERÊNCIAS}

AMIGUES, R. Trabalho do professor e trabalho de ensino. In: MACHADO, A. R. (Org.). $O$ ensino como trabalho: uma abordagem discursiva. Londrina: Eduel, 2004.

CHIROKE, S. C. H. Entorno a la educación pre universitaria en el Perú. El caso de la UNMSM. Un estudio diagnóstico. Lima: CEPREUNMS, 2010.

CHOMSKY, N. O neoliberalismo tomou de assalto as universidades. 2014. Disponible en: < http://disciplinas.stoa.usp.br/mod/url/view.php?id=194681>. Acceso el 13 abril de 2015.

CLOT, Y. Trabalho e poder de agir. Tradução de Guilherme João de Freitas Teixeira e Marlene Machado Zica Vianna. Belo Horizonte: Fabrefactum, 2010.

CUNHA, D. M. Ergologia. In: OLIVEIRA, D. A.; DUARTE, A. M. C.; VIEIRA, L. M. F. DICIONÁRIO: trabalho, profissão e condição docente. Belo Horizonte: UFMG/Faculdade de Educação, CDROM, 2010.

CUNHA, D. M. Trabalho, política e saberes na perspectiva ergológica. In: XI COLÓQUIO INTERNACIONAL DE PSICOSSOCIOLOGIA E SOCIOLOGIA CLÍNICA. Anais... Belo Horizonte: Simpósio Trabalho e Política no Mundo Contemporâneo, 2010. Disponible en: <http:www.fafich.ufmg.br/>. Acceso el 15 febrero de 2012.

FAÏTA, D. La conduite du TGV: exercices de styles. Champs Visuels: Paris, n. 6, p. 75-86, 1997.

FIGUEIREDO, M.; ATHAYDE, M.; BRITO, J. (Org.). Labirintos do trabalho: interrogações e olhares sobre o trabalho vivo. Rio de Janeiro: DP\&A, 2004. 
ODDONE, I. et al. Ambiente de trabalho: a luta dos trabalhadores pela saúde. São Paulo: Hucitec, 1986.

PÁSARA, L. La mercantilización de la educación universitaria. En Palestra portal de asuntos públicos de la PUCP. 2013. Disponible en: < http:// repositorio.pucp.edu.pe/.../ mercantilizacion_educacion_Pasara.pdf>. Acceso el 20 abril de 2016.

QUIJANO, A. Lo público y lo privado: un enfoque latinoamericano. En: Cuestiones y horizontes: de la dependencia histórico-estructural a la colonialidad/descolonialidad del poder. CLACSO, Buenos Aires. 2014.

ROCA, D. F. El trabajo del profesor en una universidad peruana originaria de un movimiento estudiantil. 2017. 336 f. Tese (Doutorado em Educação) - Faculdade de Educação, Universidade Federal de Minas Gerais, Belo Horizonte, 2017.

ROCHA, D.; DAHER, M. C.; SANT'ANNA, V. L. A entrevista em situação de pesquisa acadêmica: reflexões numa perspectiva discursiva. Revista polifonia, v. 8, n. 8, 2004.

RODRíGUEZ, J. V. La Política universitaria en el Perú. In: XXVI CONGRESO DE LA ASOCIACIÓN LATINOAMERICANA DE SOCIOLOGÍA. Acta... Guadalajara: Asociación Latinoamericana de Sociología, 2007. Disponible en: https://www.aacademica.org/000066/556. Acceso el 20 abril de 2016.

SCHWARTZ, Y. A comunidade científica ampliada e o regime de produção de saberes. Trabalho e Educação, Belo Horizonte, n. 7, p. 38-46, jul./dez. 2000.

SCHWARTZ, Y. O trabalho numa perspectiva filosófica. Trad. Marie-Annik Bernier. Cuiabá: FE/UFMT, 2003.

SCHWARTZ, Y. Circulações, dramáticas, eficácias da atividade industriosa. Tradução J. Brito e M. Athayde. Trabalho, Educação e Saúde, Rio de Janeiro, v. 2, n. 1, p. 33-55, mar. 2004.

SCHWARTZ, Y. Conceituando o trabalho, o visível e o invisível. Trabalho, Educação e Saúde, Rio de Janeiro, v. 9, p. 19-45, 2011.

SCHWARTZ, Y.; DURRIVE, L. (Org.). Trabalho \& Ergologia: conversas sobre a atividade humana. 2. ed. Niterói: Editorial Universidade Federal Fluminense, 2010. 318 p.

SGUISSARDI, V. Universidade pública estatal: entre o público e privado/mercantil. Revista Educação e Sociedade, v. 26, n. 90, jan./abr. 2005. 


\section{Daniel Fabián Roca Flores Pinto}

Docente e investigador en el campo de las ciencias sociales, línea de investigación en Educación, Trabajo, Políticas Públicas y Formación Humana. Doctor en Educación por la Universidade Federal de Minas Gerais - Brasil. Magister en Docencia e Investigación en Educación Superior por la Universidad Peruana Cayetano Heredia. Especialista en Imagen y Relaciones Institucionales, Graduado en Ciencias Administrativas. Profesor universitario en pregrado y post grado; experiencia en la gestión académica y en la gestión administrativa en organizaciones del sector de los servicios.

daniel2471@yahoo.es

\section{Daisy Moreira Cunha}

Doutora em Filosofa pela Aix-Marseille Université, mestra em Educação pela Faculdade de Educação (FaE) da UFMG, graduada em Pedagogia pelo Instituto de Educação de Minas Gerais. Professora associada da FaE/UFMG, diretora do Instituto de Estudos Avançados Transdisciplinares da UFMG (IEAT/UFMG).

daisycunhaufmg@gmail.com

\section{Rogério Cunha Campos}

Doutor em História e Filosofia da Educação pela Universidade de São Paulo (USP). Professor na Faculdade de Educação da Universidade Federal de Minas Gerais (UFMG).

roge41@uol.com 